\title{
A 100-Element MODFET Grid Amplifier
}

\author{
Michael P. De Lisio*, Cheh-Ming Liu, Alina Moussessian, David B. Rutledge \\ Department of Electrical Engineering, California Institute of Technology, Pasadena, CA 91125 \\ James J. Rosenberg \\ Department of Engineering, Harvey Mudd College, Clarement, CA 91711
}

\begin{abstract}
A 100-element quasi-optical amplifier is presented. The active devices are custom-fabricated modulation-doped field-effect transistors (MODFET's). Common-mode oscillations were suppressed using resistors in the input gate leads. The grid has $9 \mathrm{~dB}$ of gain at $10.1 \mathrm{GHz}$. The 3-dB bandwidth is $1.2 \mathrm{GHz}$. We present a model for the gain of the grid versus frequency and compare measurement with theory.
\end{abstract}

\section{INTRODUCTION}

Quasi-optical amplifiers have recently become the object of considerable attention. By combining the powers of many solid-state devices in free space, these amplifiers eliminate the losses associated with waveguide or transmission-line feed networks. The approaches vary. Quasi-optical amplifiers using patch antennas [1,2], back-to-back integrated horn antennas [3,4], folded slot antennas [5], and probe antennas $[6]$ have been demonstrated. The grid amplifier $[7,8,9]$ is another approach. The grid amplifier is an array of closely-spaced differential-pairs of transistors. A 100-element grid of heterojunctionbipolar-tarnsistors (HBT's) has shown $10 \mathrm{~dB}$ gain at $10 \mathrm{GHz}[8]$. Here we report on the design and behavior of a 100-element grid amplifier with MODFET differential-pairs as the active devices.

\section{GRID AMPLifier DEsign}

The unit cell of the grid amplifier is shown in Fig. 1(a). A horizontally-polarized incident wave excites currents on the input gate leads. This drives the transistor pair in the differential mode. Currents on the output drain leads produce the amplified output beam. The thin meandering lines supply dc bias to the drain and source. The gate bias is supplied by the gate input lead.

The differential pair chips were custom-fabricated by Martin Marietta Laboratories. The layout of a chip is shown in Fig. 1(b). It consists of two pulse-doped 0.1- $\mu \mathrm{m}$ pseudomorphic MODFET's connected as a differential pair. Each transistor has a gate width of $100 \mu \mathrm{m}$. The $1-\mathrm{k} \Omega$ resistor allows the gate bias to be continuous from cell to cell. This is possible because the gates draw very little dc bias current. The $0.5-\mathrm{pF}$ capacitor and $2-\mathrm{k} \Omega$ resistor are feedback elements to help stabilize the devices. The two $100-\Omega$ resistors in the source were intended to help suppress common-mode oscillations.

The grid amplifier measurement setup is shown in Fig. 2(a). The horizontally-polarized input beam is incident from the left. The output beam is vertically-polarized and is radiated to the right. Metal-strip input and output polarizers provide independent tuning of the output and input circuits. The polarizers and grid are made on Duroid substrates with a relative dielectric constant of 2.2 .

The grid can be modelled using the transmission-line model illustrated in Fig. 2(b). For convenience, only one-half of the unit cell is used in the analysis, with the result that the characteristic impedances are half of their usual values. Free space, then, is represented by the $189-\Omega$ resistor. The various substrates and air gaps appear as transmission lines. The metal-strip polarizers appear in shunt, either as low-impedance inductors or high-impedance capacitors, depending on the polarization. The input and output leads of the grid are modelled as inductors. Numerical values for these reactive elements were computed using the method of moments to approximate the surface current distribution and then the induced emf method to determine the impedance. Finally, the meandering drain and source bias lines are modelled as a shunt inductance at the input surface of the grid. 


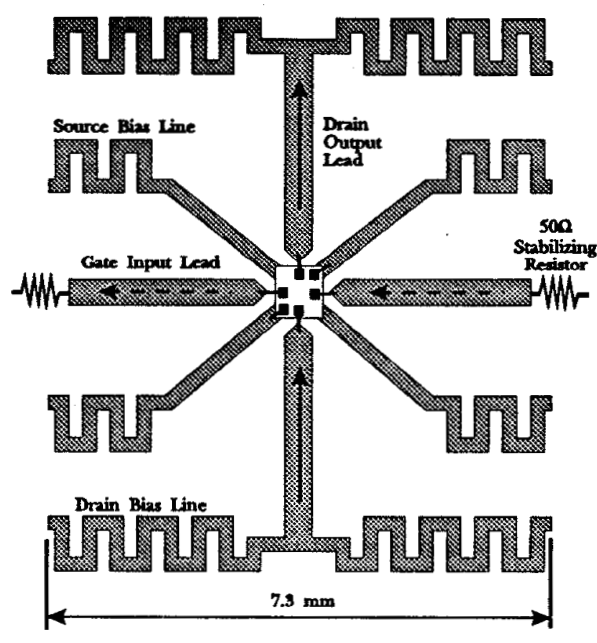

(a)

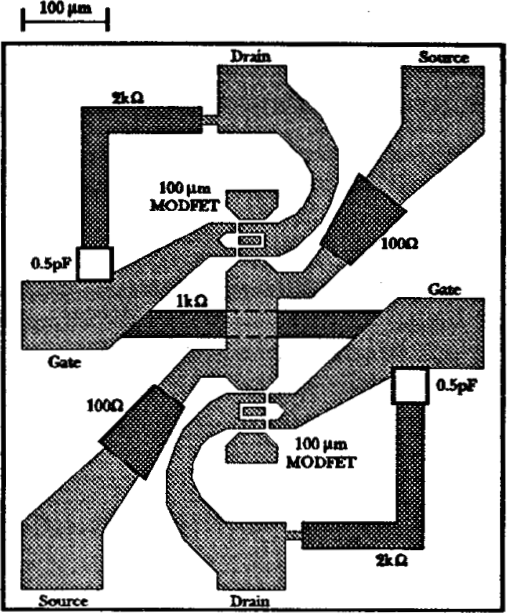

(b)

Figure 1. (a) Grid amplifier unit cell. The entire grid is a 10-by-10 array of these unit cells. (b) MODFET differential pair chip. The chips were fabricated by Martin Marietta Laboratories.

\section{iII. Grid Amplifier Performance}

Initially, the grid suffered from the common-mode oscillations similar to those detailed in [9]. The gain of the amplifier grid at the onset of oscillation was $3 \mathrm{~dB}$ at $10 \mathrm{GHz}$. The bias current was $9 \mathrm{~mA}$ per device-less than half of the recommended bias. Because of the need to bias the gate, stabilizing capacitors could not be used. Instead, $50-\Omega$ chip resistors were placed in the input gate leads of the grid, midway between the devices, as seen in Fig. 1(a). With the resistors in place, the grid could be biased to a current of over $15 \mathrm{~mA}$ per device. Theoretically, these resistors reduce the peak gain by about $2 \mathrm{~dB}$. They will also increase the noise figure considerably. In the future, it would be desirable to suppress oscillations using only reactive elements that would not have such a detrimental effect on the gain and noise performance. Preliminary investigations reveal that it should be possible to suppress the common-mode oscillations by eliminating the $100-\Omega$ resistors originally thought necessary for preventing oscillations, although this has not yet been experimentally verified.

The gain of the grid was measured using the far-field approach described in [8]. A peak gain of $9 \mathrm{~dB}$ at $10.1 \mathrm{GHz}$ was achieved with the polarizer positions shown in Fig. 2(a). A plot of gain versus frequency is shown in Fig. 3(a). The measurement agrees reasonably well with the modelled gain. The 3-dB bandwidth is $1.2 \mathrm{GHz}$. To further investigate the validity of the model, the gain was measured as a function of output polarizer position. Fig. 3(b) shows good agreement between theory and experiment. Finally, the grid amplifier gain is plotted as a function of total grid drain current in Fig. 4 .

\section{Conclusion}

A successful 100-element MODFET grid amplifier has been demonstrated. The grid has a peak gain of $9 \mathrm{~dB}$ at $10.1 \mathrm{GHz}$, with a 3 -dB bandwidth of $1.2 \mathrm{GHz}$. The results agree well with theoretical expectations. Common-mode oscillations were suppressed with resistors in the gate leads. Additional testing of this grid is underway, including noise figure, power saturation, and radiation pattern measurements. 


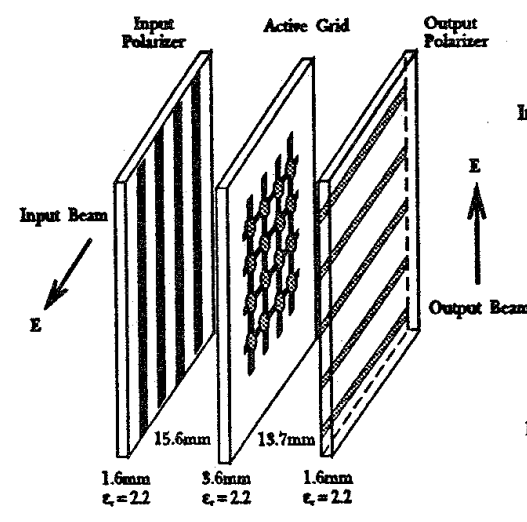

(a)

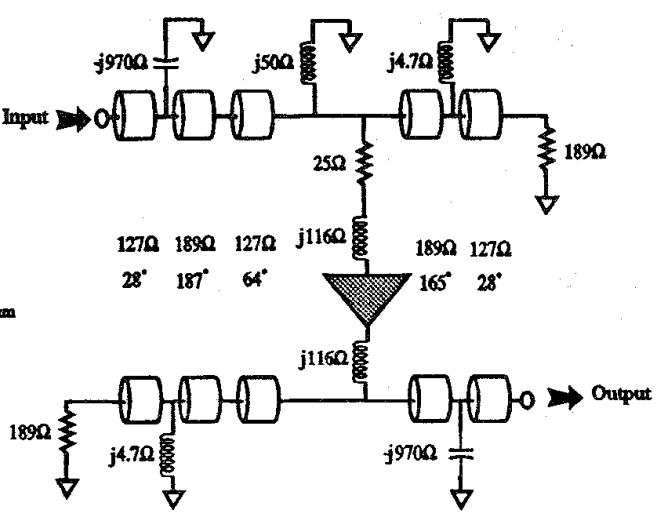

(b)

Figure 2. (a) Grid amplifier measurement setup. (b) Transmission-line equivalent circuit used for gain analysis.

\section{ACKNOWLEDGMENTS}

This research was supported by the Army Research Office and a grant from Martin Marietta Laboaratories. M.P. De Lisio holds an NSF fellowship. The authors are grateful to Martin Marietta Laboratories for supplying the differential-pair chips. Furthermore, we would like to thank Dr. S. Weinreb, Dr. S. Duncan, and Dr. N. Byer of Martin Marietta Laboratories for their suggestions and support.

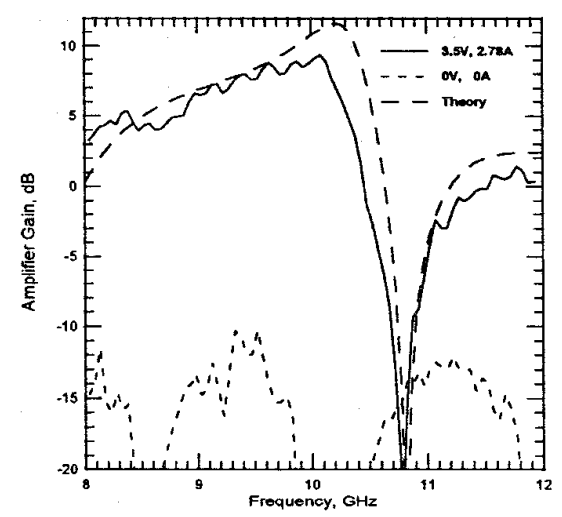

(a)

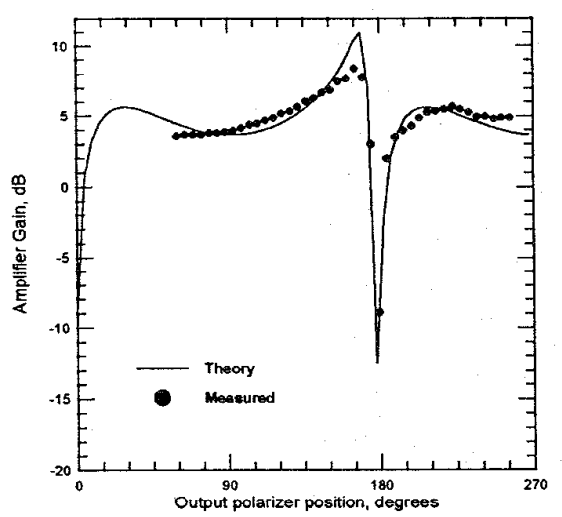

(b)

Figure 3. (a) Grid amplifier gain versus frequency. (b) Amplifier gain at $10 \mathrm{GHz}$ as a function of output polarizer position. The input polarizer was not moved. 


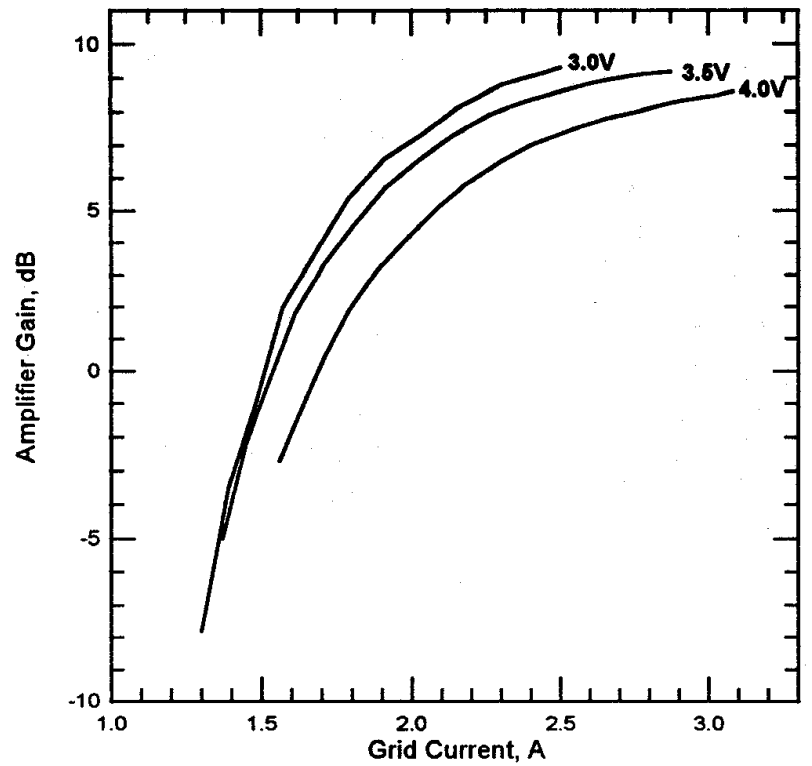

Figure 4. Grid amplifier gain at $10.1 \mathrm{GHz}$ versus total grid current. The grid current was varied under a constant grid drain voltage by adjusting the gate voltage.

\section{REFERENCES}

[1] T. Mader, J. Schonberg, L. Harmon, Z.B. Popović, "Planar MESFET Transmission Wave Amplifier," Electronics Lett., vol. 29, pp. 1699-1701, Sept. 1993.

[2] N. Sheth, T. Ivanov, A. Balasubramaniyan, A. Mortazawi, "A Nine HEMT Spatial Amplifier," 1994 IEEE MTT-S Int. Microwave Symp. Dig., pp. 1239-1242, 1994.

[3] C.-Y. Chi, G. Rebeiz, "A Quasi-Optical Amplifier," IEEE Microwave and Guided Wave Lett., vol. 3, pp. 164-166, June 1993.

[4] T.P. Budka, M.W. Trippe, S. Weinreb, G.M. Rebeiz, "A $75 \mathrm{GHz}$ to $115 \mathrm{GHz}$ Quasi-Optical Amplifier," IEEE Trans. Microwave Theory Tech., vol. 42, pp. 899-901, May 1994.

[5] H.S. Tsai, M.J.W. Rodwell, R.A. York, "Planar Amplifier Array With Improved Bandwidth Using Folded-Slots," IEEE Microwave and Guided Wave Lett., vol. 4, pp. 112-114, April 1994.

[6] N.J. Kolias, R.C. Compton, "A Microstrip-Based Unit Cell for Quasi-Optical Amplifier Arrays," IEEE Microwave and Guided Wave Lett., vol. 3, pp. 330-332, Sept. 1993.

[7] M. Kim, J.J. Rosenberg, R.P. Smith, R.M. Weikle, J.B. Hacker, M.P. De Lisio, D.B. Rutledge, "A Grid Amplifier," IEEE Microwave and Guided Wave Lett., vol. 1, pp. 322-324, Nov. 1991.

[8] M. Kim, E.A. Sovero, J.B. Hacker, M.P. De Lisio, J.-C. Chiao, S.-J. Li, D.R. Gagnon, J.J. Rosenberg, D.B. Rutledge, "A 100-Element HBT Grid Amplifier," IEEE Trans. Microwave Theory Tech., vol. 41, pp. 1762-1771, Oct. 1993.

[9] C.-M. Liu, E.A. Sovero, M.P. De Lisio, A. Moussessian, J.J. Rosenberg, D.B. Rutledge, "Gain and Stability Models for HBT Grid Amplifiers," submitted to 1995 IEEE AP-S Int. Symp., 1995. 